\title{
Good mid-term results with the trident peripheral self-locking cup: a clinical evaluation and migration measurement with EBRA
}

\author{
Dietmar Dammerer $^{1} \cdot$ Philipp Blum $^{1}$ (D) $\cdot$ David Putzer $^{2} \cdot$ Andreas Tscholl $^{1} \cdot$ Michael C. Liebensteiner $^{1} \cdot$ Martin Thaler $^{1}$
}

Received: 6 May 2020 / Accepted: 15 October 2020 / Published online: 9 November 2020

(c) The Author(s) 2020

\begin{abstract}
Introduction The most common cause of failure in total hip arthroplasty (THA) is aseptic loosening. Uncemented cup migration analysis by means of Einzel-Bild-Roentgen-Analyse (EBRA) has shown to be a good predictive indicator for early implant failure if the cup migrates more than $2 \mathrm{~mm}$ within 4 years after surgery. In this study, we performed a migration analysis of an uncemented peripheral self-locking (PSL) press-fit cup after 4 years follow-up.

Materials and methods We retrospectively reviewed all patients who received a trident PSL press-fit cup at our department between 2004 and 2017. A total of 636 patients were identified. As inclusion criteria for radiological analysis, a minimum follow-up of 2 years was defined. We reviewed medical histories and performed radiological analysis using EBRA software. EBRA measurements and statistical investigations were performed by two independent investigators.

Results A total of 149 cups in 146 patients (female 82; male 64) met our inclusion criteria. Mean age at surgery was 65 years (33-89). We found a significant improvement in the WOMAC score pre- to postoperative $(p<0.0001)$. EBRA migration analysis showed a mean total migration of $0.6 \mathrm{~mm}(0.0-8.2)$ over our follow-up period of 4 years. Of the investigated cups, $69.8 \%$ showed a migration rate smaller than $2 \mathrm{~mm}$ in the investigated follow-up.

Conclusion The acetabular cup used in our study provides low migration at final follow-up. Therefore, a good long-term outcome can be expected for the PSL cup.

Trial registration Trial registration number is 20181024-1875 and date of registration is 2018-10-24.
\end{abstract}

Keywords Cup migration · Total hip arthroplasty · Cementless · Einzel-Bild-Röntgen-Analyse (EBRA)

Philipp Blum

philippblum1@gmx.de

Dietmar Dammerer

dietmar.dammerer@tirol-kliniken.at

David Putzer

david.putzer@i-med.ac.at

Andreas Tscholl

andreastscholl@ymail.com

Michael C. Liebensteiner

michael.liebensteiner@i-med.ac.at

Martin Thaler

martin.thaler@i-med.ac.at

1 Department of Orthopaedics and Traumatology, Medical University of Innsbruck, Anichstrasse 35, 6020 Innsbruck, Austria

2 Department of Experimental Orthopedics, Medical University of Innsbruck, Sonnenburgstr. 16, 6020 Innsbruck, Austria

\section{Introduction}

The most common cause of failure in total hip arthroplasty (THA) is aseptic loosening [1]. According to the literature, a cup migration of more than $1 \mathrm{~mm}$ within the first 2 years, or more than $2 \mathrm{~mm}$ at 4 years after surgery is a well-established risk factor for early implant failure [2-5]. Previously published studies of uncemented cup migration by Einzel-Bild-Roentgen-Analyse (EBRA) have shown this to be a good predictive indicator and threshold for later aseptic loosening [6-8].

Einzel-Bild-Roentgen-Analyse is a computer-assisted method for measuring the migration of acetabular cups using standard anterior-posterior (ap) pelvic radiographs without requiring additional means at exposure (e.g. ball markers) [9]. EBRA has a proven accuracy and sensitivity in detecting migration of more than $1 \mathrm{~mm}$ as compared to RSA (roentgen stereophotogrammetric analysis) $[10,11]$. 
As THA is one of the most successful medical procedures, various implant designs are available. Peripheral self-locking designs are commonly used as an acetabular implant because the rationale behind the PSL design is to provide better primary stability at the outer acetabular rim. The cup used in this study is the Trident PSL (peripheral self-locking) by Stryker ${ }^{\circledR}$ (Stryker, Kalamazoo, Michigan, USA). According to the Australian Orthopaedic Association National Joint Replacement Registry (AOANJRR), with a total of 8450 implanted cups Trident was the most common cup used in primary total conventional hip replacement in 2018, whereby AOANJRR does not exactly distinguish between PSL and hemispherical cup type [12]. Since the Trident cup system was released in 1999 [13], it has been widely used for primary as well as revision THA. The Trident peripheral self-locking cup has a $1.8-\mathrm{mm}$ peripheral press-fit built into the shell [14]. This design is intended to allow better fixation to the peripheral lunate of the acetabulum [13]. In 2012, Nunag et al. presented preliminary data from a migration analysis of the Trident PSL with a very low number of cases. Nunag et al. [13] mentioned good clinical function of the implant, whereby migration analysis showed radiological instability in most implants within the first 2 years after surgery.

In the present study, we investigated the clinical results and migration behavior using EBRA of the uncemented Trident PSL cup with a mean follow-up of 4 years.

\section{Materials and methods}

After approval by the local ethics committee (Medical University of Innsbruck, Austria, Europe), we applied a retrospective study design and reviewed all consecutive patients who had received an uncemented Trident PSL cup in our Department between 2004 and 2017. During this time, a total of 636 Trident PSL acetabular cups were implanted as primary THA.

We investigated the medical histories for sociodemographic data, surgical approach, body mass index, cut-tosuture time, and the indication for THA. In addition, the Western Ontario and McMaster Universities Osteoarthritis (WOMAC) Index was utilized pre- and one year postoperatively as patient-reported outcome measure for function and pain [16].

Prosthetic stability and cup migration were assessed with EBRA (German: Einzel-Bild-Röntgen-Analyse) [9] from plain $\mathrm{x}$ rays. EBRA is a well-established method for evaluating standard anterior-posterior radiographs without requiring additional means at exposure (e.g. ball markers) (Fig. 1). Simulating the spatial situation, it computes parameters of longitudinal and transverse migration of prosthetic cup and femoral head. Migration of the femoral head, the acetabular

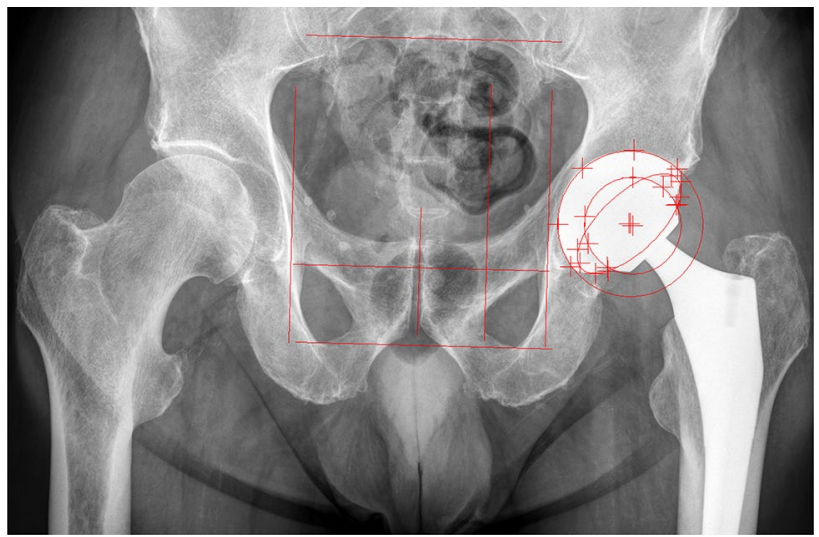

Fig. 1 Postoperative anterior-posterior x-ray of a primary total hip arthroplasty with EBRA cup software lines included

cup and the wear in the horizontal and vertical directions can be studied. A comparability algorithm using a grid of transverse and longitudinal tangents of the pelvis contour divides serial radiographs into sets of comparable ones. Migration is measured only between comparable radiographs. The $95 \%$ confidence limits for EBRA results are $1.0 \mathrm{~mm}$ for longitudinal and $0.8 \mathrm{~mm}$ for transverse migration [9]. All radiographs were taken in a strictly standardized manner according to the EBRA protocol (anterior-posterior radiographs; patient standing in an upright position; full weight-bearing) [9]. In our department we follow patients with radiographs routinely before discharge, 6 weeks after surgery, 12 months postoperative and at a consecutive annual check-up. In addition to this routine, we perform additional radiographs if the patient has any complaints, sorrows or questions concerning the THA. In our study, migration analysis with EBRA called for a minimum of four radiographs per patient and a minimum radiological follow-up of 2 years. Cup migration analysis was performed by one independent investigator, who was not involved in the surgeries or postoperative patient treatment. All patients in the study group fulfilled the EBRA criteria. The head and cup sizes used for EBRA calibration were taken from the intraoperative protocol. Cup flowchart is shown as Fig. 2.

In addition, as suggested in the literature, our patient cohort was split into two groups to measure the cup size effect: patients with an implanted cup size $\geq 54 \mathrm{~mm}$ and patients with a cup size $<54 \mathrm{~mm}[15,16]$.

\section{Statistics}

Mean, median, range, and standard deviation were calculated for the various measurement parameters. For the analysis, Access and Excel (Microsoft Office Professional Plus 2010, Redmond, WA, USA) as well as Graph Pad Prism (Version 


\section{Enrollment}

\section{Allocation}

Assessed for eligibility $(n=636)$
Excluded $(n=68)$

- Not follow-up over 24 months



Fig. 2 CONSORT guidelines cup flow diagram

8.0, GraphPad Software, Inc., La Jolla, CA, USA) were used. WOMAC scores were compared using the Wilcoxon's signed-rank test. Total migration was calculated with the Pythagorean theorem expressing the length of the vector. We defined loosening as a total migration of more than $2 \mathrm{~mm}$ within 4 years [6]. For comparison of the total migration divided by cup size the non-parametric Mann-Whitney $U$ test was used. A $p$ value of 0.05 was considered statistically significant.

\section{Results}

A total of 149 cups in 146 patients (female 82; male 64) fulfilled our inclusion criteria. In three patients the Trident PSL cup was implanted on both sides. At the time of surgery, the patient mean age was 65 years (33-89) and mean body mass index was $26.8 \mathrm{~kg} / \mathrm{m}^{2}$ (15.2-39.4). Mean follow-up was 4 years (2.0-9.8). Pre-operative diagnosis was osteoarthritis in 130 hips (87.3\%), avascular necrosis of the femoral head in nine $(6.0 \%)$, hip dysplasia and secondary osteoarthritis in nine $(6.0 \%)$ and femoral neck fracture in one hip
(0.7\%). Mean cut-to-suture time was $72 \mathrm{~min}$ (33-188). The cup sizes most frequently used in our study were $52 \mathrm{~mm}$ (17.4\%) and $56 \mathrm{~mm}(17.4 \%)$. The mean cup inclination was $42.3^{\circ}\left(24.1^{\circ}-61.3^{\circ}\right)$ and the mean cup anteversion was $18.5^{\circ}$ $\left(4.3^{\circ}-48.5^{\circ}\right)$. Further details are shown in Tables 1 and 2.

We found a significant improvement in total WOMAC score from 57 (4-99) pre-operatively to 17 (0-95) 1 year postoperatively $(p<0.0001)$. No further significant differences were found for the subgroups (stiffness, etc.) of the WOMAC score.

Migration analysis at 4 years follow-up was calculated for 43 of the 149 cups with an EBRA given comparability limit of $3.0 \mathrm{~mm}(95 \% \mathrm{CI})$. Negative horizontal migration values were defined as medial migration. Negative vertical migration (distal migration) up to $1 \mathrm{~mm}$ was caused by the limited accuracy of the EBRA measurement method. A complete set of radiographs at every point of time (e.g. 1-2 years, etc.) was not available for each cup in our study. This resulted in a different number of cases at the corresponding migration behavior analysis over time.

Based on Krismer et al.'s [6] definition of aseptic loosening, seven $(8.1 \%)$ of 86 cups showed a migration of more than 
Table 1 Demographics of study patients

\begin{tabular}{ll}
\hline Number of patients & 82 \\
Female & 64 \\
Male & 146 \\
Total & $65(33-89)$ \\
Mean age (years) & $26.8(15.2-39.4)$ \\
BMI (kg/m $\left.{ }^{2}\right)$ & $72(33-188)$ \\
Cut-to-suture time (min) & \\
Surgical approach & 146 \\
Direct anterior approach & 1 \\
Direct anterior approach with release of the mus- \\
$\quad$ culus tensor fasciae latae & 2 \\
Missing data & \\
Surgical position & 149 \\
Supine & \\
Preoperative diagnosis & 130 \\
Osteoarthritis & 9 \\
Avascular necrosis of the femoral head & 9 \\
Hip dysplasia & 1 \\
Fractures of the femoral neck &
\end{tabular}

Range is given in brackets

Table 2 Details of implanted components

\begin{tabular}{cl}
\hline $\begin{array}{l}\text { Trident PSL cup size (mm) } \\
(\%)\end{array}$ & $3(2.0)$ \\
\hline 46 & $8(5.4)$ \\
48 & $28(18.8)$ \\
50 & $26(17.4)$ \\
52 & $25(16.8)$ \\
54 & $26(17.4)$ \\
56 & $22(14.8)$ \\
58 & $4(2.7)$ \\
60 & $6(4.0)$ \\
62 & $1(0.7)$ \\
64 & \\
Head size (mm) (\%) & $30(20.1)$ \\
28 & $100(67.1)$ \\
32 & $19(12.8)$ \\
s36
\end{tabular}

$1 \mathrm{~mm}$ after 2 years. Of the 149 cups, 43 had sufficient EBRA follow-up to determine migration behavior after 4 years. Of these 43 cups, 13 (30\%) migrated more than $2 \mathrm{~mm}$. We found that nearly $70 \%$ of the investigated cups showed a migration rate smaller than $2 \mathrm{~mm}$ in the investigated follow-up period of 4 years. Table 3 and Fig. 3 show details of migration behavior after 1-4 years. Percentages of migrated cups are given in Table 4.

While investigating the cup size effect, we found no statistically significant difference in total migration between the two sub-cohorts: cup size $<54$ and $\geq 54 \mathrm{~mm}$ at 1 year $(p=0.9672), 2$ years $(p=0.1830), 3$ years $(p=0.4199)$ and 4 years $(p=0.2379)$ radiological follow-up.

During our follow-up period, four cups had to be revised. All four were due to a periprosthetic infection after 27, 34, 46 and 90 months. No further revisions were required. Six patients died during our clinical follow-up, but not because of surgical or postoperative reasons.

\section{Discussion}

To the best of our knowledge, the present study includes the longest follow-up period and the largest number of uncemented peripheral self-locking cups investigated by means of EBRA to date. As THA is one of the most successful medical procedures, various implant designs are available. Peripheral self-locking designs are commonly used as acetabular implant because the rationale behind the PSL design is to provide better primary stability at the outer acetabular rim. We found the investigated cup to have a very low mean total migration of $0.6 \mathrm{~mm} /$ year during our follow-up period of 4 years. Previously published and applied threshold values for cup loosening show that our results are well in line with the current literature. In our study, nearly $70 \%$ of the cups showed migration to be less than $2 \mathrm{~mm}$ within 4 years. From previously published data we can expect the TridentPSL cup to have excellent long-term survival.

It is assumed that primary stability is a prerequisite for bony ingrowth of the acetabular component. For this purpose, EBRA is a suitable method for identifying and measuring the migration behavior of THA components [9, 17, 18]. While roentgen stereometric analysis (RSA) is considered the gold standard for migration measurements, Abrahams et al. [10] recently reported good agreement between EBRA cup and RSA measurements of migration of acetabular cups in THA. Knowing that and applying a retrospective study design, we used EBRA cup software for our investigations of migration behavior.

Table 3 Mean total migration in millimetres ( $\mathrm{mm}$ ) over time

\begin{tabular}{lllll}
\hline & 12 months $(n=137)$ & 24 months $(n=86)$ & 36 months $(n=108)$ & 48 months $(n=43)$ \\
\hline Migration of the trident PSL (mm/year) & $0.1(0.0-1.7)$ & $0.4(0.0-2.0)$ & $0.8(0.0-1.1)$ & $1.5(0.0-8.2)$ \\
\hline
\end{tabular}

Range is given in brackets 


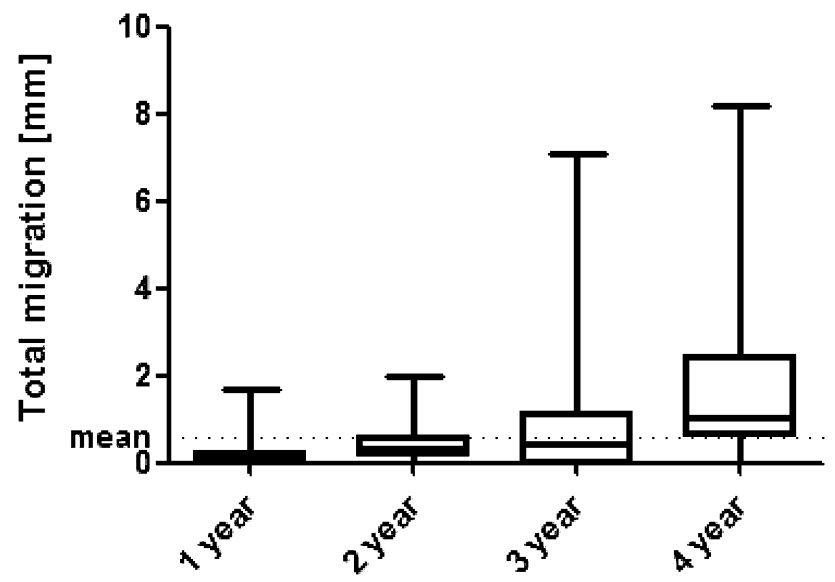

Fig. 3 Boxplots showing mean migration and bars showing the minimum and maximum migration for radiological follow-up

Table 4 Total migration in millimetres $(\mathrm{mm})$ over time

\begin{tabular}{lllll}
\hline & $\begin{array}{l}\text { 1 year } \\
(n=137)\end{array}$ & $\begin{array}{l}2 \text { years } \\
(n=86)\end{array}$ & $\begin{array}{l}3 \text { years } \\
(n=108)\end{array}$ & 4 years $(n=43)$ \\
\hline$\leq 1$ & $135(98.5 \%)$ & $79(91.9 \%)$ & $76(70.4 \%)$ & $20(46.5 \%)$ \\
$>1$ & $2(1.5 \%)$ & $6(7.0 \%)$ & $21(19.4 \%)$ & $10(23.3 \%)$ \\
$>2$ & $0(0.0 \%)$ & $1(1.1 \%)$ & $5(4.6 \%)$ & $10(23.3 \%)$ \\
$>3$ & $0(0.0 \%)$ & $0(0.0 \%)$ & $3(2.8 \%)$ & $1(2.3 \%)$ \\
$>4$ & $0(0.0 \%)$ & $0(0.0 \%)$ & $1(0.9 \%)$ & $0(0.0 \%)$ \\
$>5$ & $0(0.0 \%)$ & $0(0.0 \%)$ & $2(1.9 \%)$ & $2(4.6 \%)$ \\
\hline
\end{tabular}

A study by Nunag et al. [13] gives a mean total migration of $1.5 \mathrm{~mm}$ for the Trident PSL cup after 2 years follow-up. Assuming the above-mentioned threshold for significant migration of an uncemented cup, the authors stated in their study that $54 \%$ of their cups migrated more than $1 \mathrm{~mm}$ and another $23 \%$ more than $2 \mathrm{~mm}$ in the 2-year follow-up [13]. In comparison to the results reported by Nunag et al., we found in our study a significantly smaller rate of cup migration. Our results show that $8.1 \%$ of the investigated cups migrated more than $1 \mathrm{~mm}$ in 2 years. While in our study $13(30.2 \%)$ cups migrated more than $2 \mathrm{~mm}$ after 4 years, only 7 (16.3\%) of these cups showed a further migration of more than $1 \mathrm{~mm}$ compared to the previous measurement. Although our EBRA migration analysis covered a period of 4 years after surgery, as compared with Nunag's 2-year follow-up period [13], our mean migration rate of $1.5 \mathrm{~mm}$ at 4 years was similar the rate published by Nunag et al. [13]. The patient sample studied by Nunag et al. was smaller, showed a higher cup migration rate and a mean observed radiolucent gap of $1.4 \mathrm{~mm}$ between cup and bone in two-thirds of all investigated cases [13]. In contrast to the surgical technique published by the manufacturer, Nunag et al. reported that the actually reamed cavity was on average $1 \mathrm{~mm}$ smaller than the reamer [13]. The instruction manual for the surgical technique recommends reaming line to line.

Several factors and the influence of migration were reported in previous studies. Especially the effect of cup size on migration yielded different results. While Stihsen et al. found a significantly greater migration for cups $\geq 54 \mathrm{~mm}$, Takatori et al. reported a negative correlation between cup size and distance of migration $[15,16]$. Stihsen et al. [15] suspected that the soft bone surrounding the larger cup provides a bigger surface for movement, which may lead to increased migration. As we found no statistical differences in total migration between cup size $<54$ and $\geq 54 \mathrm{~mm}$ at $1-4$ years radiological follow-up, we can confirm the findings of Stoeckl et al. [19], namely that there is no correlation between cup size and migration. Stoeckl et al. investigated and published the migration behaviour of the Duraloc cup '100 Series' (DePuy Synthes, Warsaw, IN, USA) after 2 and 4 years follow-up. Using the same criteria for migration analysis, $48 \%$ of the Duraloc cups showed significant migration and a mean total migration rate of $1.13 \mathrm{~mm}$ at 2 years [19]. However, the reduction in migration speed after 4 years observed by Stoeckl et al. [20] provided a better result for the Duraloc cup than the initially surveyed migration rate after 2 years had suggested.

This study has several limitations including the retrospective methodology and the absence of a control group. In addition, other factors that might influence migration behavior of the cup, such as comorbidities and under-, line-toline- or over-reaming, were not investigated. Furthermore, only 149 (23.4\%) of 636 implanted cups were eligible for this study due to a lack of follow-up or insufficient number of x-rays. This may increase the susceptibility of the study to selection bias. Moreover, there was a varying number of radiographs and duration of follow-up for each hip. This may have influenced the migration results due to the smoothing function of the software. Based on the published EBRA-cup threshold by Krismer et al. [6], none of our cups have been revised during the observed follow-up period. Therefore, long-term results are necessary to observe the performance and survival of the investigated cup. Additionally, further studies are needed to state more clearly the relevance of the EBRA-cup threshold by Krismer et al. [6], in short- to mid-term migration behavior studies. Finally, the EBRA cup software uses the horizontal line that can be labeled on the pubic symphysis or the ischial foramen as the reference segment for proximal translation, while assuming that the pelvis is in continuity and is a single reference segment.

In conclusion, we show a low migration rate for a peripheral self-locking cup with excellent clinical results up to 4 years after surgery. From the results of the migration analysis, we can predict good long-term survival for the cup. Further investigation is needed for cups that showed a migration of more than $2 \mathrm{~mm}$ at 4 years follow-up. 
Author contributions All listed authors have substantially contributed to this study. All authors have reviewed and confirmed the accuracy of the whole manuscript.

Funding Open access funding provided by University of Innsbruck and Medical University of Innsbruck. This research did not receive any specific grant from funding agencies in the public, commercial, or not-for-profit sectors.

Availability of data and material Data will be sent if necessary.

Code availability Not applicable.

\section{Compliance with ethical standards}

Conflict of interest Author Martin Thaler has received research grants from Stryker which are not related to the content of the study. Martin Thaler is Treasurer of the European Hip Society. Dietmar Dammerer, Philipp Blum, David Putzer, Andreas Tscholl and Michael C. Liebensteiner declare that they have no conflict of interest.

Ethics approval The local ethics committee approved the study.

Consent to participate Informed consent was obtained from all participants.

Consent for publication All authors have reviewed and confirmed the accuracy of the whole manuscript.

Open Access This article is licensed under a Creative Commons Attribution 4.0 International License, which permits use, sharing, adaptation, distribution and reproduction in any medium or format, as long as you give appropriate credit to the original author(s) and the source, provide a link to the Creative Commons licence, and indicate if changes were made. The images or other third party material in this article are included in the article's Creative Commons licence, unless indicated otherwise in a credit line to the material. If material is not included in the article's Creative Commons licence and your intended use is not permitted by statutory regulation or exceeds the permitted use, you will need to obtain permission directly from the copyright holder. To view a copy of this licence, visit http://creativecommons.org/licenses/by/4.0/.

\section{References}

1. Johnsen SP, Sorensen HT, Lucht U, Soballe K, Overgaard S, Pedersen AB (2006) Patient-related predictors of implant failure after primary total hip replacement in the initial, short- and long-terms. A nationwide Danish follow-up study including 36,984 patients. J Bone Joint Surg Br 88:1303-1308. https://doi.org/10.1302/0301620X.88B10.17399

2. Stocks GW, Freeman MA, Evans SJ (1995) Acetabular cup migration. Prediction of aseptic loosening. J Bone Joint Surg Br 77:853-861

3. Ilchmann T, Kesteris U, Wingstrand H (1998) EBRA improves the accuracy of radiographic analysis of acetabular cup migration. Acta Orthop Scand 69:119-124. https://doi.org/10.3109/17453 679809117610

4. Ilchmann T, Markovic L, Joshi A, Hardinge K, Murphy J, Wingstrand H (1998) Migration and wear of long-term successful Charnley total hip replacements. J Bone Joint Surg Br 80:377381. https://doi.org/10.1302/0301-620x.80b3.8455
5. Wyss T, Kagi P, Mayrhofer P, Notzli H, Pfluger D, Knahr K (2013) Five-year results of the uncemented RM pressfit cup clinical evaluation and migration measurements by EBRA. J Arthroplasty 28:1291-1296. https://doi.org/10.1016/j.arth.2012.11.004

6. Krismer M, Stock1 B, Fischer M, Bauer R, Mayrhofer P, Ogon M (1996) Early migration predicts late aseptic failure of hip sockets. J Bone Joint Surg Br 78:422-426

7. Ilchmann T, Neher S, Maurer F, Weise K (2007) Modes of failure of a threaded acetabular cup : a radiographic study with EBRA of 42 revised cups. Int Orthop 31:211-216. https://doi.org/10.1007/ s00264-006-0155-2

8. Meek RM, Michos J, Grigoris P, Hamblen DL (2002) Mid-term results and migration behaviour of a ti-alloy cemented stem. Int Orthop 26:356-360. https://doi.org/10.1007/s00264-002-0386-9

9. Krismer M, Bauer R, Tschupik J, Mayrhofer P (1995) EBRA: a method to measure migration of acetabular components. J Biomech 28:1225-1236

10. Abrahams JM, Callary SA, Jang SW, Hewitt J, Howie DW, Solomon LB (2020) Accuracy of EBRA-cup measurements after reconstruction of severe acetabular defects at revision THR. J Orthop Res. https://doi.org/10.1002/jor.24623

11. Biedermann R, Krismer M, Stockl B, Mayrhofer P, Ornstein E, Franzen H (1999) Accuracy of EBRA-FCA in the measurement of migration of femoral components of total hip replacement. Einzel-Bild-Rontgen-Analyse-femoral component analysis. J Bone Joint Surg Br 81:266-272. https://doi. org/10.1302/0301-620x.81b2.8842

12. Registry AOANJR (2020) Annual Report 2019

13. Nunag P, Deakin AH, Oburu E, Sarungi M (2012) Two-year radiologic assessment of the trident peripheral self-locking cup using EBRA. Hip Int 22:511-515. https://doi.org/10.5301/ HIP.2012.9744

14. Dammerer D, Putzer D, Glodny B, Petersen J, Arrich F, Krismer M, Biedermann R (2018) Occult intra-operative periprosthetic fractures of the acetabulum may affect implant survival. Int Orthop. https://doi.org/10.1007/s00264-018-4084-7

15. Stihsen C, Pabinger C, Radl R, Rehak P, Windhager R (2008) Migration of the Duraloc cup after 5 years. Int Orthop 32:791794. https://doi.org/10.1007/s00264-007-0405-y

16. Takatori Y, Ninomiya S, Umeyama T, Yamamoto M, Moro T, Nakamura K (2002) Bipolar revision arthroplasty for failed threaded acetabular components: radiographic evaluation of cup migration. J Orthop Sci 7:467-471. https://doi.org/10.1007/s0077 60200081

17. Ilchmann T, Franzen H, Mjoberg B, Wingstrand H (1992) Measurement accuracy in acetabular cup migration. A comparison of four radiologic methods versus roentgen stereophotogrammetric analysis. J Arthroplasty 7:121-127. https://doi.org/10.1016/08835403(92)90004-a

18. Phillips NJ, Stockley I, Wilkinson JM (2002) Direct plain radiographic methods versus EBRA-digital for measuring implant migration after total hip arthroplasty. J Arthroplasty 17:917-925. https://doi.org/10.1054/arth.2002.34529

19. Stockl B, Sandow M, Krismer M, Biedermann R, Wimmer C, Frischhut B (1999) Migration of the Duraloc cup at 2 years. J Bone Joint Surg Br 81:51-53. https://doi. org/10.1302/0301-620x.81b1.9036

20. Stoeckl B, Brabec E, Wanner S, Krismer M, Biedermann R (2005) Radiographic evaluation of the Duraloc cup after 4 years. Int Orthop 29:14-17. https://doi.org/10.1007/s00264-004-0600-z

Publisher's Note Springer Nature remains neutral with regard to jurisdictional claims in published maps and institutional affiliations. 